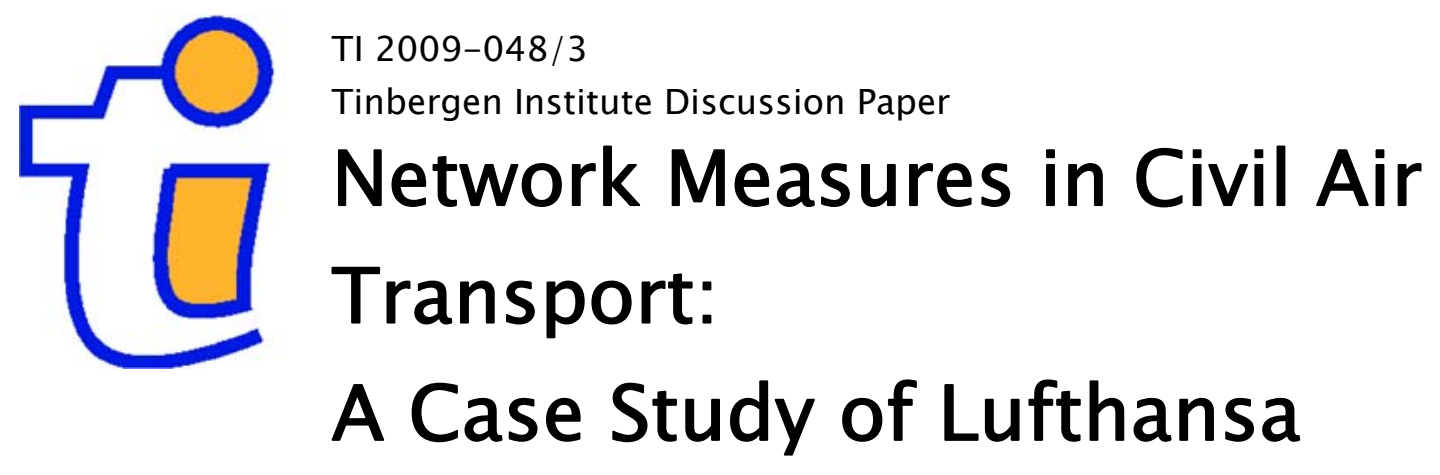

Aura Reggianil
Sara Signorettil
Peter Nijkamp
Alessandro Cento 3

' University of Bologna, Italy;

2 VU University Amsterdam, The Netherlands, and Tinbergen Institute;

${ }^{3}$ KLM Royal Dutch Airlines, Milan, Italy. 


\section{Tinbergen Institute}

The Tinbergen Institute is the institute for economic research of the Erasmus Universiteit Rotterdam, Universiteit van Amsterdam, and Vrije Universiteit Amsterdam.

Tinbergen Institute Amsterdam

Roetersstraat 31

1018 WB Amsterdam

The Netherlands

Tel.: +31(0)205513500

Fax: $+31(0) 205513555$

Tinbergen Institute Rotterdam

Burg. Oudlaan 50

3062 PA Rotterdam

The Netherlands

Tel.: + $31(0) 104088900$

Fax: $+31(0) 104089031$

Most TI discussion papers can be downloaded at http://www.tinbergen.nl. 


\title{
Network Measures in Civil Air Transport: A Case Study of Lufthansa
}

\author{
Aura Reggiani, ${ }^{\text {a }}$ Sara Signoretti, ${ }^{\text {a }}$ Peter Nijkamp, ${ }^{\text {b }}$ Alessandro Cento ${ }^{\mathrm{c}}$
}

\begin{abstract}
Air transport networks have exhibited a trend towards complex dynamics in recent years. Using Lufthansa's networks as an example, this paper aims to illustrate the relevance of various network indicators - such as connectivity and concentration - for the empirical analysis of airline network configurations. The results highlight the actual strategic choices made by Lufthansa for its own network, as well in combination with its partners in Star Alliance.
\end{abstract}

Keywords: air transport, complex networks, connectivity, concentration, Lufthansa.

\section{Introduction}

Network analysis has already a long history in operations research and quantitative social science research. In the past, much attention has been paid to shortestroute algorithms (for example, the travelling salesman problem), where the spatial configuration of networks was put in the centre of empirical investigation. Integer programming, linear and nonlinear programming turned out to offer a proper analytical toolbox. In recent years, we have seen several new trends, in particular, the rise of hub-and-spoke systems in liberalized networks, the emergence of dynamic adjustments to new competitive conditions and the increase in complexity in international networks.

Furthermore, it appears that in the past decades many social, spatial and economic systems show an organized pattern characterized by network features, such as transportation, telecommunication, information or energy systems. As a consequence, much attention has recently been paid to the study of network properties

\footnotetext{
${ }^{a}$ Aura Reggiani, Sara Signoretti

Department of Economics, Faculty of Statistics, University of Bologna, Italy, email: aura.reggiani@unibo.it,sara98@supereva.it

${ }^{\mathrm{b}}$ Peter Nijkamp

Department of Spatial Economics, Faculty of Economics and Business Administration, VU University Amsterdam, The Netherlands, email: pnijkamp@feweb.vu.nl

${ }^{c}$ Alessandro Cento

KLM Royal Dutch Airlines, Milan, Italy, email: Alessandro.Cento@klm.com
} 
emerging in many social, spatial and economic fields, as witnessed by the vast amount of literature published in the past years (Barthélemy 2003; Gorman and Kulkarny 2004; Gorman 2005; Schintler et al. 2005a,b; Reggiani and Nijkamp 2006; Patuelli 2007). Air transport is a prominent example of modern network constellations and will be addressed in this paper from a connectivity perspective.

Air transport shows indeed clear network features, which impact on the way single airline carriers operate (Button and Stough 2000). The abundant scientific literature on airline networks has addressed this topic in terms of theoretical modelling and empirical measurements on different typologies of airline network configurations. This strand of recent research aimed to measure the network structure in relation to the effects of: (a) the market deregulation in United States in 1978 and in the European Union in the 1990s, (b) new trends in recent airline business strategies denoted as 'low cost' principles. Low cost carriers developed rather fast after the deregulation policy, by acquiring a competitive network advantage on traditional airlines, which consequently seemed to reorganise rapidly their airline network to respond to the new market dynamics.

In this context, interesting research has emerged that mainly addressed the issue of describing and classifying networks by means of geographical concentration indices of traffic or flight frequency (Caves et al. 1984; Toh and Higgins 1985; McShan 1986; Reynolds-Feighan 1994, 1998, 2001; Bowen 2002; Lijesen 2004; Cento 2006). These measures, such as the Gini concentration index or the Theil index, provide a proper measure of frequency or traffic concentration of the main airports in a simple, well-organized network. However, if a real-world network structure is complex, including multi-hub or mixed point-to-point and hub-spokes connections, the concentration indices may record high values for all types of structure, but fail to clearly discriminate between different network shapes (Alderighi et al. 2007). There is a need for a more appropriate measurement of connectivity structures in complex networks.

Starting from the above considerations and research challenges, the present paper aims to investigate the scientific potential and applicability of a series of network connectivity/concentration indices, in order to properly typify and map out complex airline network configurations. Specifically, these various network indicators will be adopted and tested to describe the main properties - in terms of the network connectivity and configuration - of Lufthansa's airline system. The aims of the present paper are then: (a) to detect the extent to which the real network configuration is close to typical network models that evolved over time; (b) to examine how concentration measures can point to the different network topologies; and (c) to study the way nodes are connected, that is, to analyse their distribution function.

The present article is organized as follows: Section 2 will provide a brief description of the main models of network connectivity that have been developed in the framework of (spatial or social) network analysis. In this section, the focus will mainly be on the concept of vertex degree distribution in a network and on the main indicators used for the analysis of air transport. Next, Section 3 presents a novel empirical analysis of Lufthansa's network; the methods provided by net- 
work analysis are applied in order to understand more thoroughly the real network's topology. Finally, Section 4 will offer some conclusions from the present paper, as well as some further research challenges.

\section{Network Models and Measures in Air Transport Systems}

\subsection{Preface}

Many economic activities are currently characterized by network characteristics with a high degree of complexity, since their processes and outcomes depend not only on the choices of the single agents but also on the dynamic - often nonlinear - interactions between them in a continuous dynamic interplay (Reggiani and Nijkamp 2006 ${ }^{1}$ ). A clear example of a complex spatial-economic network is the geographical network of the air transport industry: understanding its peculiarities and responding to these features can bring about substantial advantages for both consumers and producers (Button and Stough 2000). Airline network analysis has gained much popularity in recent years.

Modelling complex networks is also a great challenge: on the one side, the topology of the network is governing the complex connectivity dynamics (see, for instance, Barabási and Oltvai 2004); on the other side, the functional-economic relationships in such networks might also depend on the type of connectivity structure. The understanding of these two interlinked network aspects may be instrumental for capturing and analysing airline network patterns. Starting from the above considerations, we will review, in the next subsection, the main connectivity models and measures which have recently gained a great deal of attention in the scientific literature, with a particular view to air transport networks.

\subsection{Network Models}

In the last decades network theory has gained scientific interest and sophisticated network models have been used in different fields, including economics and geography (Waters 2006). This trend faced also quite some difficulty, because existing models were not able to clearly describe the network properties of many real-

${ }^{1}$ These authors point out that the main feature of complexity is that the outcome (of the activity of a complex system) 'should not be obvious from the single building blocks' (Bossomaier and Green 2000). Consequently, the term complexity indicates that the final result cannot be foreseen even when the single components of a system are known and studied. 
world systems, whose complexity could not fully be understood (Barabási and Albert 1999).

Spatial-economics systems - including air transport networks - are complex, because agents interact, obtaining significant benefits by means of a joint activity (Boschma 2005). This interacting process may become a permanent feature thus leading to a new meso- or macro structure, for example, to the creation of clusters.

Air transport systems have over the past years been experiencing such clustering processes. An example is provided by airlines' alliances. ${ }^{2}$ The main reason why airline carriers cooperate of aggregate stems from cost reductions they can thus obtain. Being a member of an alliance impacts on the carriers' strategy for a long time and also influences the network configuration they adopt. It is worth noteworthy that alliances play also an important role in determining market dynamics; in 2005, the three main alliances in air transport accounted for 80 per cent of the total capacity offer. ${ }^{3}$ Therefore, we need to develop airline network models that can adequately take into account clustering and merger processes.

A further important trend many real networks show is the so-called 'SmallWorld (SW) effect'. This term indicates that the diameter ${ }^{4}$ of a network is so short that it takes only a few movements along links in order to move between any two nodes of a network (Reggiani and Vinciguerra 2007). In air transport systems, we can point out the SW effect by taking into consideration and comparing the network configuration of single carriers or of alliances; such systems exhibit a clear SW effect when it takes only a small number of flights to link the two most distant airports in the network.

Alongside the SW effect, the SW network model has been developed in order to take into account both the SW effect and the related clustering processes (Watts and Strogatz 1998). The main features of this model are a short diameter and a high clustering coefficient.

A further elaboration of the SW model is the so called Scale-Free (SF) network introduced by Barabási and Albert (1999) in order to incorporate two mechanisms upon which many real networks have proven to be based: growth and preferential attachment. The former points to the dynamic character of networks, which grow by the addition of new nodes and new vertices; the latter explains how new nodes enter the network, namely by connecting themselves to the nodes having the highest number of links.

An important feature of SF networks is represented by their vertex degree distribution ${ }^{5} P(k)$ which is proportional to $k^{-\gamma}$ (with $k$ being the number of links), that is, to a power law. The value of the degree exponent $\gamma$ depends on the attrib-

${ }^{2}$ The processes underlying the creation of an alliance can be clearly depicted by considering the integration of Lufthansa and Swiss, described in the Lufthansa Annual Report (2005); available on the website http://konzern.lufthansa.com/en/html/ueber_uns/swiss/index.html).

${ }^{3}$ See http://www.tourismfuturesintl.com/special\%20reports/alliances.html.

${ }^{4}$ The concept of a diameter will be defined in Subsection 2.3 .

${ }^{5} P(k)$ is the probability that a chosen node has exactly $k$ links (Barabási and Oltvai 2004). See also Eq. (1). 
utes of the single systems and is crucial to detect the exact network topology, in particular the existence of the hubs (highly connected nodes). As Barabási and Oltvai (2004) highlight, a SF network embeds the proper hub-and-spoke model only when $\gamma=2$, while for $2<\gamma \leq 3$ a hierarchy of hubs emerge. For $\gamma>3$, the hub features are absent and the SF network behaves like a random one.

In air transport systems, we can point out SW networks by considering fullservice carriers. Without national or political impediments in a free market, these carriers typically organize their network into a hub-and-spoke system, where one or a few central airports called 'hubs' have a high number of links to the other airports called 'spokes'. Passengers travelling from a place of origin to a place of destination have to stop typically in one or a few hubs to change aircraft. Hubs are organised in order to allow flight connectivity by coordinating the scheduled timetable of the arriving and departing flights. Investigating the airline strategy in designing hub connectivity and timetable coordination has been the aim of several empirical network studies. Some examples of theoretical and empirical investigation of hub connectivity can be found in the works of Bootsma (1997), Dennis (1998), Rietveld and Brons (2001), Veldhuis and Kroes (2002), and Burghouwt and de Wit (2003). As a consequence, the hub has to manage normally a high volume of traffic at the same time, due to their central connecting role in the network.

In contrast to SF networks, we have to highlight also random networks (Erdös and Rényi 1959), which display homogeneous, sparse patterns, without cluster characters. Their vertex degree distribution follows a Poisson distribution. ${ }^{6}$

In air transport, random networks are useful to map point-to-point connections, as it is the case for low-cost airlines (Cento 2006). In the ideal point-to point network all airports are connected to each other, so that passengers can fly from one airport to any other directly without stopping in any hub to change aircrafts. These networks have a low diameter, as a consequence of the high number of direct links between airports. Reggiani and Vinciguerra (2007, p. 148) point out that a random network can be seen as 'a homogeneous system which gives accessibility to the majority of the nodes in the same way'. Furthermore, as it is evident by looking at the plot of the exponential function, the probability to find highly connected nodes is equal to 0 . Therefore, no clear hubs exist, and the network configuration appears to be random because no single airport displays a dominant role in a connected network.

In the next Subsection 2.3, we will address two main degree (connectivity) distributions that have often been observed in empirical experiments, vis-à-vis exponential and power-law.

${ }^{6}$ For a review of random models, SW models and SF models, see Albert and Barabási (2002) and Joeng (2003). 


\subsection{Network Degree Distributions}

The vertex degree distribution is one of the key tools we may use to point out the network configuration (Reggiani and Vinciguerra 2007), since this function determines the way nodes are connected. It can be defined as the probability $P(k)$ of finding nodes with $k$ links.

In general, we can state that:

$P(k)=N(k) / N$,

where $N(k)$ is the number of nodes with $\mathrm{k}$ links and $N$ is the number of nodes of the network.

With regard to the network topologies developed in the framework of graph theory, complex systems tend to show two main degree distributions: the Poisson distribution (Erdös and Rényi 1959) and the power-law function (Barabási and Bonabeau 2003).

The former is defined as:

$P(k) \square e^{<k>} \frac{<k>^{k}}{k !}$,

and describes networks - so-called random networks - where the majority of nodes have approximately the same number of links, close to the average degree $<k>$ (Barabási and Albert 1999). Eq. (2) is a distinctive feature of point-to-point networks, such as those adopted by low-cost airlines; this network topology is typical of equilibrated economic-geographical areas, where a high number of direct links can be profitably operated.

The power-law function is defined as:

$P(k) \square k^{-\gamma}$,

and characterizes networks having a small number of nodes with a very high degree while the majority of nodes have a few links. Eq. (3) has important economic implications: it characterizes SF networks, where the term SF refers to the fact that 'the power-law distribution does not change its form no matter what scale is used to observe it' (Reggiani and Vinciguerra 2007, p. 150), and that, in these networks, distances are irrelevant. Therefore, we expect to find SF networks in 'global networks', such as the Internet and air transport, and in general in those networks where relevant economic aggregation clusters (preferential attachments) attract flows from distant nodes.

It interesting to note that from the above distribution functions (Eqs 2 and 3) we can extrapolate the related cost/utility/impedance functions (Reggiani and Vinciguerra 2007). However, when the identification of the two functions is ambigu- 
ous, we need to obtain additional information from network theory (for example, centrality indices, dominance indices). A multidimensional approach is needed in this respect, where not only the way airports are connected is relevant (spatial network components), but also the geometrical architecture of the network, as well as its degree of network homogeneity (physical network components).

In the subsequent Subsections 2.4 and 2.5 we will now introduce some indicators and measures we can adopt to study the network configuration of carriers: the computation of the above indices is crucial to understand the tendency to agglomeration of concentration patterns, and hence the possibility of hierarchical network relations among nodes.

It is moreover important to identify a SF network because of its strong features in terms of robustness and vulnerability. In the case of a random attack (or disturbance) on nodes, the SF network will strongly persist, because a random attack will probably damage nodes that have only a few connections, which are the majority. Nevertheless in case of an attack against the main hubs, the network will easily be fragmented. Consequently, we might also talk of 'vulnerability/permeability' of the SF network: if a strategic input, for example, a virus, is dispersed in the hubs, it is certainly diffused all over the network. On the other hand, random networks are weak against a random attack which will cause the split of the network.

Consequently, it is important to identify hubs in the network in order to prevent targeted attacks and to preserve the system (Gorman 2005). The identification of such characteristics is certainly useful to the understanding of the dynamics of air network configurations, also from the perspective of policy/planning interventions.

\subsection{Network Topology Indices}

Airline networks may exhibit simple or complex topologies. Networks have been given several definitions in the framework of graph theory, as for instance by Harary (1969): 'a network is a graph, or directed graph, together with a function which assigns a positive real number to each edge'. In this context it is useful to outline the geometrical indicators most frequently used to represent the network shape; they are illustrated in Table 1.

It should be mentioned that the first three indices measure the centrality of a vertex in a graph, while the last two can be used to investigate the networks' topological properties (Reggiani and Vinciguerra 2007). It is necessary to underline that the 'geodesic distance', used to compute closeness, betweenness and diameter, represents the shortest of all distances between two nodes (Freeman 1979).

In the context of our empirical experiments, we will apply the above indicators to explore Lufthansa's network structure and configuration, since all complex systems characterized by a network structure share properties exclusively depending on network's configuration (see also Waters 2006). Before starting our empirical 
analysis, Subsection 2.5 will illustrate additional indices that we may use to investigate the networks' concentration.

Table 1 Network's topology indices

\begin{tabular}{|c|c|c|c|c|}
\hline $\begin{array}{l}\text { Index or } \\
\text { measurement }\end{array}$ & Description & Formulation & Variables & Source \\
\hline Degree & $\begin{array}{l}\text { The degree of a } \\
\text { node is given by } \\
\text { the number of its } \\
\text { links }\end{array}$ & $k(v)$ & $\begin{array}{l}k(v) \text { is the number of } \\
\text { links of node } v\end{array}$ & $\begin{array}{l}\text { Barabási } \\
\text { and Oltvai } \\
(2004)\end{array}$ \\
\hline Closeness & $\begin{array}{l}\text { It indicates a } \\
\text { node's proximity } \\
\text { to the other nodes }\end{array}$ & $C(v)=\frac{1}{\sum_{t \in V} d_{v t}}$ & $\begin{array}{l}d_{v t} \text { is the shortest path } \\
\text { (geodesic distance) } \\
\text { between nodes } v \text { and } t ; n \\
\text { is the number of nodes in } \\
\text { the network }\end{array}$ & $\begin{array}{l}\text { Newman } \\
(2003)\end{array}$ \\
\hline Betweenness & $\begin{array}{l}\text { It indicates a } \\
\text { node's ability to } \\
\text { stand between the } \\
\text { others, and } \\
\text { therefore, to } \\
\text { control the flows } \\
\text { among them }\end{array}$ & $B(v)=\sum_{s \neq t \neq v \in V} \frac{\sigma_{s t}(v)}{\sigma_{s t}}$ & $\begin{array}{l}\sigma_{s t}(v) \text { and } \sigma_{s t} \text { are, } \\
\text { respectively, the number } \\
\text { of geodesic distances } \\
\text { between } s \text { and } t \text { that pass } \\
\text { through node } v \text {, and the } \\
\text { overall number of } \\
\text { geodesic distances } \\
\text { between nodes } s \text { and } t\end{array}$ & $\begin{array}{l}\text { Freeman } \\
(1977)\end{array}$ \\
\hline Diameter & $\begin{array}{l}\text { It measures the } \\
\text { maximum value of } \\
\text { the geodesic } \\
\text { distances between } \\
\text { all nodes }\end{array}$ & $D=\max _{s, t \in V, s \neq t} d_{s t}$ & $\begin{array}{l}d_{s t} \text { is the geodesic } \\
\text { distance between nodes } s \\
\text { and } t\end{array}$ & $\begin{array}{l}\text { Boccaletti } \\
\text { et al. } \\
(2006)\end{array}$ \\
\hline $\begin{array}{l}\text { Clustering } \\
\text { coefficient }\end{array}$ & $\begin{array}{l}\text { It measures the } \\
\text { cliquishness of a } \\
\text { node }\end{array}$ & $C l(v)=\frac{l_{v}}{\max l_{v}}$ & $\begin{array}{l}l_{v} \text { and }{ }_{\text {max }} l_{v} \text { are, } \\
\text { respectively, the number } \\
\text { of existing and maximum } \\
\text { possible links between } \\
\text { the nodes directly } \\
\text { connected to node } v \text { (its } \\
\text { neighbours) }\end{array}$ & $\begin{array}{l}\text { Watts and } \\
\text { Strogatz } \\
(1998)\end{array}$ \\
\hline
\end{tabular}

\subsection{Network Concentration Indices}

If we want to detect the networks' configuration (random versus SF) we also need to understand to what extent these networks are concentrated, because the existence of hubs implies a high degree of concentration (Reynolds-Feighan 2001). To 
6 Aura Reggiani, Sara Signoretti, Peter Nijkamp, Alessandro Cento

this purpose we will use: a) the Gini concentration index; b) the Freeman centrality index $;{ }^{7}$ and c) the entropy index. These three indices are illustrated in Table 2.

Table 2 Network's concentration indices

\begin{tabular}{|c|c|c|c|c|}
\hline Indicator & Formula & Use & Variables used & Sources \\
\hline $\begin{array}{l}\text { Gini } \\
\text { concentration } \\
\text { index }\end{array}$ & $G=\frac{\sum_{i=1}^{n} \sum_{j=1}^{n}\left|x_{i}-x_{j}\right|}{2 n^{2} \mu}$ & $\begin{array}{l}\text { It is a measure } \\
\text { of } \\
\text { geographical } \\
\text { concentration }\end{array}$ & $\begin{array}{l}x_{i,} x_{j} \text { are the number of } \\
\text { weekly flights from } \\
\text { airports } i \text { and } j, \text { ranked } \\
\text { in increasing order; } n \text { is } \\
\text { the number of airports in } \\
\text { the network; } \mu \text { is } \\
\sum_{i} x_{i} / n\end{array}$ & $\begin{array}{l}\text { Cento } \\
(2006)\end{array}$ \\
\hline $\begin{array}{l}\text { Freeman } \\
\text { centrality } \\
\text { index }\end{array}$ & $F_{B}=\frac{\sum_{i}\left[F_{B}\left(x^{*}\right)-F_{B}\left(x_{i}\right)\right]}{n^{3}-4 n^{2}+5 n-2}$ & $\begin{array}{l}\text { It is a measure } \\
\text { of similarity to } \\
\text { a perfect star } \\
\text { network }\end{array}$ & $\begin{array}{l}F_{B}\left(x_{i}\right)=\sum \sum b_{j k}\left(x_{i}\right) \text { is } \\
\text { the } j<k j<k \\
\text { betweenness centrality } \\
\text { of node } x_{i} ; F_{B}\left(x^{*}\right) \text { is the } \\
\text { highest betweenness } \\
\text { centrality value of the } \\
\text { distribution }\end{array}$ & $\begin{array}{l}\text { Cento } \\
(2006)\end{array}$ \\
\hline $\begin{array}{l}\text { Entropy } \\
\text { function }\end{array}$ & $E=-\sum_{i j} p_{i j} \ln p_{i j}$ & $\begin{array}{l}\text { It measures } \\
\text { the degree of } \\
\text { spatial } \\
\text { organisation } \\
\text { and variety in } \\
\text { a system }\end{array}$ & $\begin{array}{l}p_{i j} \text { is the probability of a } \\
\text { link between nodes } i \text { and } \\
j\end{array}$ & $\begin{array}{l}\text { Nijkamp } \\
\text { and } \\
\text { Reggiani } \\
\text { (1992); } \\
\text { Frenken } \\
(2006)\end{array}$ \\
\hline
\end{tabular}

The first index $G$ measures the inequality existing in a distribution, and ranges between 0 and 1; the higher its value, the more uneven is the distribution (Freeman 1979). The second index $F$ takes into account the structure of the system, and measures the network shape as the degree of inequality in a network with respect to a perfect star network (Freeman 1979).

The third is the entropy function $E$, which shows the degree of variety existing in an economic or spatial network (Frenken 2006). In particular, entropy can be employed as a tool for studying spatial differentiation, that is, heterogeneity in a system: 'for instance, by investigating whether certain spatial configurations are completely arbitrary and disordered or whether these configurations show a cer-

${ }^{7}$ The concept of concentration aims at discerning whether or not the activity we are studying is located homogeneously over a geographical area, without considering the form of corresponding system. In the framework of our experiments, networks are concentrated to the extent that some nodes have a share of flights which is higher than the area they occupy (Freeman 1979).

The concepts of centrality - referring to single nodes - and centralization - referring to a whole network - are closely related: a network is centralized when a node, or a group of nodes, can control the flows the network represents and are consequently given higher centrality values (Freeman 1977). We can, therefore, state that centralized networks are always concentrated as well, while the opposite does not always holds. 
tain degree of spatial organization or regularity' (Nijkamp and Reggiani 1992, pp. 18-19). Therefore the entropy function indicates how organized a system is: the higher is the value of $E$, the more diversified the network (Frenken 2006).

Next, in Section 3 we will carry out an empirical study on four networks based on Lufthansa's airline network - by means of the analytical tools previously described.

\section{An Empirical Application to Lufthansa's Airline Network}

\subsection{The Data Base}

This section will focus on the geographical analysis of Lufthansa's aviation network in the year 2006. The airline network measurement is essential for exploring the airline behaviour and its implications for the supply, the traffic demand, the airports' infrastructure and aviation planning. The airline network can be subdivided into domestic, international or intercontinental configurations depending on whether the airports connected are located within a country, a continent or in different continents. Furthermore, an airline network can be interconnected or interlined to partner's networks within the alliance concerned. This classification is based on geographical, air transport-political and economic characteristics, such as airlines' degree of freedom from the Chicago Convention (see Cento 2006) market liberalization, or costs and traffic demand. Therefore, the overall network configuration is the result of the integrated optimisation of the domestic, international, and intercontinental parts of the total network. These sub-network configurations may range from fully-connected or point-to-point to hub-and-spokes configurations to alliances (fully-contracted) or to a mix of these configurations. Within this conceptual framework, we will position our analysis of four sub-networks of Lufthansa. As summarized in Table 3, we coin networks A1 and A2, referring respectively to the flights operated by Lufthansa in Europe and in the whole world, while networks B1 and B2 take into consideration - respectively at a European and at a global level - the flights operated by all the carriers which are members of Star Alliance (to which Lufthansa belongs). ${ }^{8}$

The variable under analysis is represented by the number of direct connections of each airport in the summer season of the year 2006, measured on a weekly basis. The networks are represented in Figure B1, B2 and B3 in Annex B.

${ }^{8}$ The Star Alliance member carriers are currently: Air Canada; Air New Zealand; ANA; Asiana Airlines; Austrian; bmi; LOT Polish Airlines; Lufthansa; Scandinavian Airlines; Singapore Airlines; South African Airlines; Spanair; Swiss; TAP Portugal; THAI; United Airlines; US Airways; VARIG (the list was retrieved from www.staralliance.com). 
Table 3 Lufthansa's network constellation (2006)

\begin{tabular}{lllcr}
\hline Network & $\begin{array}{l}\text { Area under } \\
\text { consideration }\end{array}$ & $\begin{array}{l}\text { Carrier or alliance operating the } \\
\text { flight }\end{array}$ & $\begin{array}{l}\text { Nodes } \\
\text { links }\end{array}$ \\
\hline A1 & Europe & Lufthansa & 111 & 522 \\
A2 & World & Lufthansa & 188 & 692 \\
B1 & Europe & Star Alliance & 111 & 3230 \\
B2 & World & Star Alliance & 188 & 6084 \\
\hline
\end{tabular}

In all four cases we only consider those airports where Lufthansa operates with its fleet and not by partner's airlines. When we consider A1 and A2 networks, we clearly see that the majority of Lufthansa's flights are operated at a continental level. On the contrary, nearly half of Star Alliance's flights are operated outside Europe. This finding is not surprising, if we consider that the carriers making up Star Alliance are mainly from non-European countries.

Subsections 3.2, 3.3 and 3.4 will now illustrate the empirical results of our experiments, aiming at analysing the connectivity and concentration patterns in the above mentioned networks.

\subsection{Lufthansa's Network Geometry}

On the basis of the indicators illustrated in Table 1, we will now show the results emerging from the related applications to the four Lufthansa's network domains $\mathrm{A} 1, \mathrm{~A} 2, \mathrm{~B} 1$ and B2. In particular, since all the indicators displayed in Table 1 characterise the nodes in a network, we will investigate by means of these indicators - in our four networks - the single nodes' features as well as the relations among nodes.

More specifically, in order to examine the nodes' location, we have computed the three centrality measures (degree, closeness and betweenness) described in Table 1. Concerning the investigation of the nodes' relations, we have examined the diameter and the clustering coefficient of the network (see again Table 1).

The degree of a node (Table 1) can be seen as a measure of centrality if we assume - in the framework of our analysis - that the best connected airports have a greater power over the whole network, as they can control a considerable amount of all flights. In all networks we find that the airports of Frankfurt and Munich have always the highest degree (see Table A1 in Annex A).

A further analysis of nodes' centrality focuses on their 'ease-of-access' to the other nodes. ${ }^{9}$ In order to investigate this concept we have computed the closeness

\footnotetext{
${ }^{9}$ It can be assumed that access to the network is easier when nodes are closer (Freeman 1979).
} 
centrality ${ }^{10}$ (Table 1). The values of this index for the networks under consideration (listed in Table A2 in the Annex) show that the highest values usually correspond to the best connected nodes; therefore, closeness centrality is able to map out - in the framework of our study - the most important airports in terms of connectivity. A similar trend can be observed by considering betweenness centrality (Table 1; the values for networks A1, A2, B1 and B2 are listed in Table A3 in Annex A). This finding is not surprising, since hubs - in the framework of the huband-spoke model - are chosen from those airports falling among the highest possible number of pairs of other airports (O'Kelly and Miller 1994; Button and Stough 2000).

The networks' topology can also be explored by examining how the various nodes relate and link, since this last attribute impacts the configuration of the whole structure. For this purpose we have computed the clustering coefficient (defined in Table 1; the ten highest values for the nodes of the four networks of our experiments are listed in Table A4 in the Annex). The values indicate a significant difference between the networks $\mathrm{A} 1$ and $\mathrm{A} 2$ and the networks $\mathrm{B} 1$ and $\mathrm{B} 2$; in the former case the airports of Frankfurt and Munich dominate the chart; in the latter case, other airports appear to emerge, thus showing that flights are spread more equally on the whole network.

In addition, we will also consider the diameter of the above networks in order to investigate how the links' patterns influence the ability to move inside the network. Both A1 and A2 have a diameter of 4, while B1 and B2 have a diameter of 2. This can be justified only if there is no significant difference in the geographical configuration between A1 and A2, approximately a hub-and spoke, while B1 and B2 can be a mixture of hub-and-spoke and point-to-point networks. In other words, the integration of Lufthansa network in the Star Alliance reduces the travel distance, as the passengers can benefit from more connections and thus shorter paths to travel between the origin and the destination. This has important implications in the context of our study, because it entails that Lufthansa's networks shrink, when we consider the flights of all Star Alliance members.

Having examined now Lufthansa nodes' characteristics, we will explore Lufthansa's network features, in particular its network concentration and connectivity. The related results will be offered in the following Subsections 3.3 and 3.4.

\subsection{Lufthansa's Network Concentration}

The study of the networks' degree of concentration - which is carried out in the present subsection - is crucial in order to detect the exact network topology, because the hub-and-spoke model is highly concentrated, while point-to-point networks do not show this feature.

${ }^{10}$ We compute the closeness centrality, as well as the subsequent betweenness centrality, using the Pajek software (http://vlado.fmf.uni-lj.si/pub/networks/pajek/). 
First, Table 4 presents the normalized Gini index (see Table 1) for the four networks under consideration. Both Star Alliance networks are less concentrated than the Lufthansa counterparts, meaning that when we enlarge the measurement to a broader network including intercontinental destinations and partners' networks, the configuration will probably evolve into a mix of multi hub-and-spoke and point-to-point structures. In particular, network A2 appears to be the most concentrated.

Table 4 Normalized Gini index

\begin{tabular}{ll}
\hline Network & Gini index \\
\hline A1 & 0.762 \\
A2 & 0.813 \\
B1 & 0.524 \\
B2 & 0.600 \\
\hline
\end{tabular}

The information provided by the Gini index refers to the degree of concentration existing in a network, without any evidence on how this concentration impacts on the network topology. For this last purpose the Freeman centrality index (Table 1) has been computed. Its normalized values are represented in Table 5. This index assumes the value 1 for a hub-and-spoke network, and the value 0 for a point-to-point network (Cento 2006).

Table 5 Normalized Freeman index

\begin{tabular}{ll}
\hline Network & Freeman index \\
\hline A1 & 0.504 \\
A2 & 0.757 \\
B1 & 0.059 \\
B2 & 0.056 \\
\hline
\end{tabular}

According to the Freeman index, again networks A1 and A2 turn out to be the most concentrated ones. In particular, A2 network seems to be again the closest to the hub-and-spoke model; we may suppose that this network is characterized by a strong hierarchy among nodes.

Finally, concerning the last concentration index, that is, entropy (Table 1), Table 6 shows the related values for the networks A1, A2, B1 and B2. The results from Table 6 show that the entropy values are higher when we consider those flights operated by Lufthansa's partners (networks B1 and B2). A likely explanation for this increase is given by the process of construction of these networks, obtained by the addition of flights to the nodes of $\mathrm{A} 1$ and $\mathrm{A} 2$, respectively. Both $\mathrm{B} 1$ and B2 are therefore the 'sum' of the networks implemented by the different carri- 
ers that are members of Star Alliance, and hence they are not the result of a specific strategy, as is the case for A1 and A2. Clearly, the above values indicate that $\mathrm{A} 1$ and A2 networks are more concentrated and less dispersed than the B1 and B2 networks; more specifically, A1 appears to be the most concentrated network.

Table 6 Entropy values

\begin{tabular}{ll}
\hline Network & Entropy \\
\hline A1 & 5.954 \\
A2 & 6.194 \\
B1 & 7.790 \\
B2 & 8.389 \\
\hline
\end{tabular}

In conclusion, from the above three indicators, networks A1 and A2 appear to be the most concentrated. However, among these two networks, A2 seems the most concentrated with respect to two indicators (Gini and Freeman), while A1 seems the most concentrated with respect to the entropy index.

In order to formally detect hub-and-spoke models, our next step will be the analysis of the vertex connectivity distribution functions of the four networks A1, A2, B1 and B2, in the light of their performance indicators (see also Section 2.3).

\subsection{Lufthansa's Network Configuration}

In Section 2.3 we have already stressed the importance of the vertex degree distribution function, in order to detect the most plausible network configuration. In this section, we will explore whether the variable 'number of weekly connections' is rank-distributed - over A1, A2, B1 and B2 - according to either an exponential or a power function.

The $R^{2}$ values and the $b$ coefficients of the two interpolating functions (exponential and power) concerning the four ranked distributions (in log terms) are listed in Table 7 . The plots of both functions for the four networks under consideration are displayed in Figs 1 and 2. 
Table 7 Exponential and power fitting of rank distributions

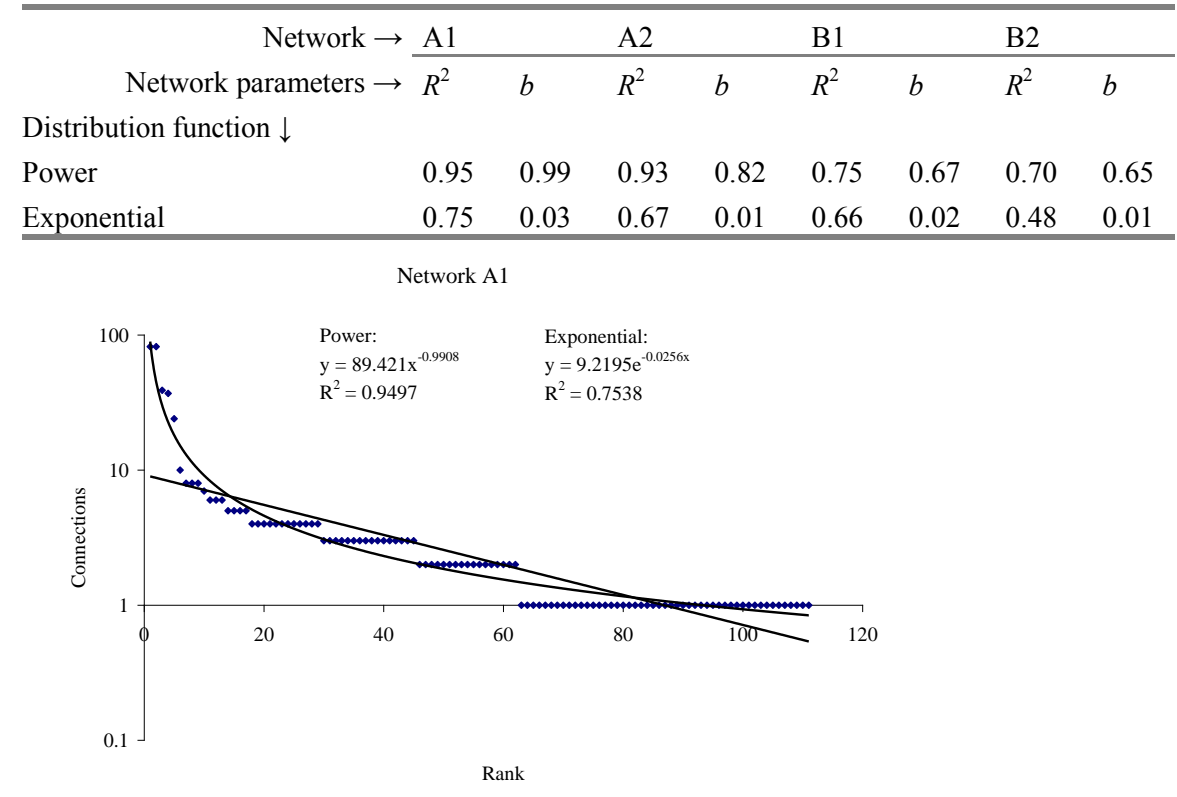

Network A2

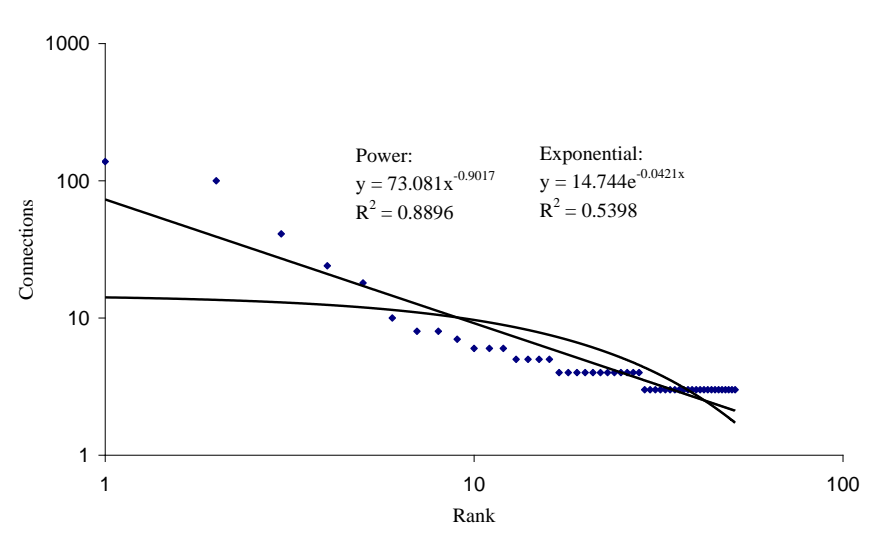

Fig. 1 Rank distribution fitting for networks A1 and A2

Both Table 7 and Figures 1 and 2 highlight that our data sets better fit a power function, as the higher $R^{2}$ values indicate. It is worth noting that the $b$ coefficient of the power function for A1, A2, B1 and B2 is respectively equal to $0.99,0.82$, 0.67 and 0.65 . If we carry out a transformation ${ }^{11}$ of these coefficients, we observe

${ }^{11}$ Adamic (2000) shows that the power-law exponent $\gamma$ (emerging from the nodes' probability distribution (Eq. 3)) is related to the power function coefficient $b$ (emerging from the distribu- 
that the A1 network displays a power-law exponent equal to 2, thus indicating a stronger tendency to a hub-and-spoke system according to Barabási and Oltvai (2004), while the other three networks A2, B1 and B2 display power-law exponent between 2 and 3, thus indicating a tendency to a hierarchy of hub/agglomeration patterns.
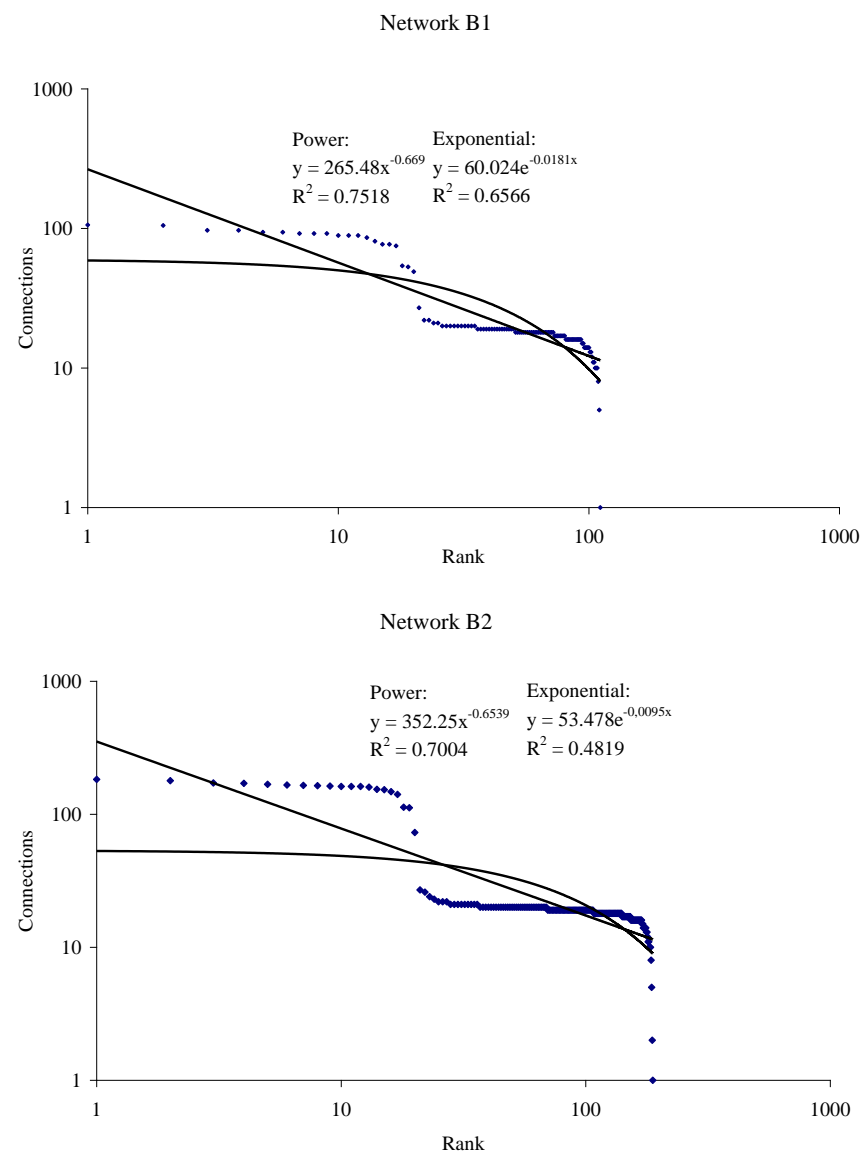

Fig. 2 Distribution fitting for networks B1 and B2

A further issue concerns the fitting of the exponential function. Also in this case we obtain high $R^{2}$ values, although inferior to the ones emerging in the power case; however, the coefficient of the exponential function is always very low, ranging from 0.01 to 0.03 (Table 7).

tion relating the degree of the nodes to their rank (rank size rule); see Figs 1 and 2) as follows: $\gamma=1+(1 / b)$ 
Therefore, if we look at the $R^{2}$ indicators, all networks under consideration appear to be in a 'border-line' situation (that is, an ambiguity between a power and exponential fitting). Nevertheless, if we look at the coefficient values, the four networks seem to show a tendency toward an agglomeration structure of SF type, expressed by a clear power-law vertex degree distribution, with the degree exponent $\gamma$ equal to 2 (network A1), or varying between 2 and 3 (networks A2, B1, B2).

A further consideration concerns the plots of networks B1 and B2 (Fig. 2). We can clearly see that both identify a power function with a cut-off. Thus, if we eliminate - in both networks B1 and B2 - those nodes which have less than 10 links, we slightly improve the fitting of their power function, obtaining for networks B1 and B2 respectively $R^{2}$ values of 0.84 and 0.75 , but still lower than the $R^{2}$ values regarding $\mathrm{A} 1$ and $\mathrm{A} 2$.

In conclusion, from the estimation results displayed in Table 3, the networks A1, A2 appear to show the strongest characteristics of concentration and preferential attachment. In particular, network A1 appears to be the closest to the hub-andspoke model, from the perspective of Barabási and Oltvai's approach. Given these preliminary results, it is worth to examine these configurations, by exploring further indicators of the network concentration, such as those defined in Sections 2.4 and 2.5. Consequently, a multidimensional method, such as Multi Criteria Analysis (MCA), taking into account - by means of an integrative approach - all adopted indicators and related results, was next carried out and applied. ${ }^{12}$ The alternatives are the four networks A1, A2, B1, B2 under consideration, while the criteria have been grouped according to three macro-criteria: network concentration, topology and connectivity (Table 8). It should be noted that, concerning the topology criteria, we have considered the diameter and the clustering coefficient, since these two indices provide the network geometry's features (see Section 3.2). In particular, concerning the latter, the average clustering coefficient has been adopted (Barabási and Oltvai 2004).

Table 8 Alternatives and criteria

\begin{tabular}{ll}
\hline Alternatives & A1 (Lufthansa, Europe) \\
& A2 (Lufthansa, World) \\
& B1 (Star Alliance, Europe) \\
& B2 (Star Alliance, World) \\
& Gini index \\
'Concentration' criteria & Freeman index \\
& Entropy \\
'Topology' criteria & Diameter \\
& Average Clustering Coefficient \\
\hline
\end{tabular}

${ }^{12}$ In particular, the Regime method and software has been used (Hinloopen and Nijkamp 1990). 


\begin{tabular}{ll}
\hline 'Connectivity' criteria & $R^{2}$ of the fitted power function (ranked degree distribution) \\
& Coefficient of the power function \\
& $R^{2}$ of the fitted exponential function (ranked degree distribution) \\
& Coefficient of the exponential function
\end{tabular}

The first group of macro-criteria is related to the networks' concentration. It should be noted that in our MCA procedure, the entropy indicator needs to be transformed positively because the real values of the entropy function increase when networks are more heterogeneous, that is, less concentrated. ${ }^{13}$ The second group of macro-criteria refers to the networks' physical measurement. Here, the diameter needs to be converted in utility, because its value is higher when networks are less centralized. The third group of macro-criteria is related to connectivity. This property is investigated through the interpolation of the ranked degree distributions, where - in the power function - the highest exponent of 0.99 implies a value of the exponent degree ${ }^{14}$ - in the associated power-law distribution - close to 2 (perfect hub-and-spoke). The $R^{2}$ and the coefficient of the exponential function need to be converted to utility, since both values indicate random and homogeneous patterns.

We have carried out five scenarios by considering: (a) all the criteria mentioned above; (b) each macro-criteria separately; (c) concentration and topology criteria together. In each scenario an equal weight, that is, unknown priority, has been given to the single criteria. The results are listed in Table 9.

Table 9 Findings of multi-criteria analyses

\begin{tabular}{llllll}
\hline $\begin{array}{l}\text { Criteria } \\
\text { considered }\end{array}$ & $\begin{array}{l}\text { All criteria } \\
\text { combined }\end{array}$ & $\begin{array}{l}\text { Concentration } \\
\text { criteria }\end{array}$ & $\begin{array}{l}\text { Topology } \\
\text { criteria }\end{array}$ & $\begin{array}{l}\text { Connectivity } \\
\text { criteria }\end{array}$ & $\begin{array}{l}\text { Concentration and } \\
\text { topology criteria }\end{array}$ \\
\hline $\begin{array}{l}\text { Hierarchy of the } \\
\text { alternatives }\end{array}$ & A1 & A2 & B1 & A1 & A1 \\
& B2 & A1 & B2 & B1 & B1 \\
& B1 & B1 & A1 & A2 & A2 \\
& B2 & A2 & B2 & B2 \\
\hline
\end{tabular}

These findings point out that network A1 prevails, however with two exceptions. The former is represented by network A2, which is the top-scorer when we consider the criteria related to the networks' concentration/geography: this finding comes from the higher centralization and concentration degree of network A2, as demonstrated by the Freeman and Gini indices. The latter exception is represented

13 The relation between concentration and centralization is described in Footnote 6 in Section 2.5 .

${ }^{14}$ See Footnote 12. 
by network B1, which prevails when we consider the criteria related to the physical measurement of networks.

It turns out that the Lufthansa network A1 is the most connected one; we can conjecture that $\mathrm{A} 1$ is close to a hub-and-spoke system, according to the values expressed by its exponent degree in the power-law distribution (see Table 7). This result confirms the dual-hubs network strategy advocated by the German carrier (Lufthansa 2005). Frankfurt and Munich act as central hubs, where all intercontinental flights depart and arrive in conjunction with the European and domestic flights. This timetable coordination is designed to allow passengers to transfer from one flight to another for different national and international destinations. The general conclusions of the present article are included in the Section 4.

\section{Conclusions}

Airline networks are fascinating examples of emerging complex and interacting structures, which may evolve in a competitive environment under liberalized market conditions. They may exhibit different configurations, especially if a given carrier has developed a flanking network framework together with partner airlines.

The present paper has investigated the network structure of four networks of Lufthansa by considering several indicators concerning the concentration, topology and connectivity (degree distribution) functions characteristics of this carrier. An integrated multidimensional approach, in particular multicriteria analysis has been adopted, in order to take into account all information obtained by the above indicators.

The related results point out that all the four Lufthansa networks can be properly mapped into the SF model of the Barabási type. In particular, network A1 can be formally identified as a hub-and-spoke structure. In general, we can conjecture a 'tendency' towards a hubs' hierarchy or hub-and-spoke configuration in Lufthansa's European network, as also witnessed by the emergence of various nodes (Frankfurt, Munich and Dusseldorf) which are organized as hubs in the framework of Lufthansa's activities. All in all the four networks exhibit a hierarchical structure mainly dominated by German airports.

The results obtained thus far highlight various characteristic features of complex aviation networks, but need to be complemented with additional investigations, in particular, on the structure and driving forces of the demand side (types of costumers, in particular). Furthermore, the market is decisive in a liberalized airline system, and hence also price responses of customers as well as competitive responses of main competitors would need to be studied in the future.

From a methodological viewpoint a refined weighted network analysis - taking into account the strength of each connecting link - might offer better insights into the topological structure of the airline network at hand (see, for example, Barrat et al. 2004). 
Acknowledgments The authors wish to thank Roberto Patuelli (Institute for Economic Research, University of Lugano) for his comments on the present chapter, as well as for his cooperation in the editing process.

\section{References}

Adamic, L.A.: Zipf, power-laws, and Pareto - A ranking tutorial. http://www.hpl.hp.com. Accessed 16 April 2007 (2000)

Albert, R., Barabási, A.-L.: Statistical mechanics of complex networks. Rev. Mod. Phys. 74, 4797 (2002)

Alderighi, M., Cento, A., Nijkamp, P., Rietveld, P.: Assessment of new hub-and-spoke and point-to-point airline network configurations. Transp. Rev. 27, 529-549 (2007)

Barabási, L.A., Albert, R.: Emerging of scaling in random networks. Sci. 286, 509-512 (1999)

Barabási, L.A., Albert, R.: Topology of evolving networks: local events and universality. Phys. Rev. Lett. 85, 5234-5237 (2000)

Barabási, L.A., Oltvai, Z.N.: Network's biology: understanding the cell's functional organization. Nat. Rev.-Genet. 5, 101-113 (2004)

Barrat, A., Barthélemy, M., Pastor-Satorras, R., Vespignani, A.: The architecture of complex weighted networks. Proc. Natl. Acad. Sci. U.S.A. 8 March. http://www.pnas.org/cgi/doi//10.1073/pnas.0400087101 (2004)

Barthélemy, M.: Crossover from scale-free to spatial networks. Europhys. Lett. 63, 915-921 (2003)

Boccaletti, S., Latora, V., Moreno, Y., Chavez, M., Hwang, D.-U.: Complex networks: structure and dynamics. Phys. Rep. 424, 175-308 (2006)

Boschma, R.A.: Proximity and innovation. a critical assessment. Reg. Stud. 39, 61-74 (2005)

Bootsma, P.D.: Airline flight schedule development; analysis and design tools for European hinterland hubs. University of Twente, Utrecht (1997)

Bowen, J.: Network change, deregulation, and access in the global airline industry. Econ. Geogr. 78, 425-439 (2002)

Burghouwt, G., de Wit, J.: The temporal configuration of European airline networks. University of Montreal, Publication AJD-74 (2003)

Button, K., Stough, R.: Air Transport Networks: Theory and Policy Implications. Edward Elgar, Cheltenham (2000)

Caves, D.W., Christensen, L.R., Tretheway, M.W.: Economics of density versus economies of scale: why trunks and local service airline costs differ. Rand J. Econ. 15, 471-489 (1984)

Cento, A.: Challenge to the airline industry: emergence of carriers and low cost carriers. $\mathrm{PhD}$ Thesis, VU University Amsterdam (2006)

Dennis, N.P.S.: Competition between hub airports in Europe and a methodology for forecasting connecting traffic. 8th World Conference on Transport Research, Antwerp (1998)

Economides, N.: The economics of networks. Int. J. Transp. Organ. 14, 669-671 (1995)

Erdös, P., Rényi, A.: On random graphs I. Publ. Math. 6, 290-297 (1959)

Freeman, L.C.: A set of measures of centrality based on betweenness. Sociom. 40, 35-41 (1977)

Freeman, L.C.: Centrality in social networks: a conceptual clarification. Soc. Netw. 1, 215-239 (1979)

Frenken, K., Nuvolari, A.: The early development of the steam engine: an evolutionary interpretation using complexity theory. Ind. Corp. Chang. 13, 419-450 (2004)

Gorman, S.P.: Networks, Security and Complexity. Edward Elgar, Cheltenham (2005)

Gorman, S.P., Kulkarni, R.: Spatial small worlds. Environ. Plan. B 31, 273-296 (2004)

Hinloopen, E., Nijkamp, P.: Qualitative multiple criteria choice analysis: the dominant regime method. Qual. Quant. 24, 37-56 (1990)

Jeong, H.: Complex scale-free networks. Phys. A 321, 226-237 (2003) 
Lijesen, M.G.: Adjusting the Herfindahl index for close substitutes: an application to pricing in civil aviation. Transp. Res. E 40, 123-134 (2004)

Lufthansa: Annual report. http://konzern.lufthansa.com/en/html/ueber_uns/swiss/index.html (2005)

McShan, W.S.: An economic analysis of hub-and-spoke routing strategy in the airline industry. $\mathrm{PhD}$ Thesis, Northwestern University (1986)

Newman, M.E.J.: A measure of betweenness centrality based on random walks. arXiv:condmat/0309045 (2003)

Nijkamp, P., Reggiani, A.: Interaction, Evolution and Chaos in Space. Springer-Verlag, Berlin (1992)

O' Kelly, E.M., Miller, H.J.: The hub network design problem: an overview and synthesis. J. Transp. Geogr. 2, 31-40 (1994)

Official Airline Guides (OAG): Worldwide flight guide. Reed Publications. http://www.oag.com. (2006)

Patuelli, R.: Regional labour markets in Germany: statistical analysis of spatio-temporal disparities and network structures. PhD Thesis, VU University Amsterdam, September (2007)

Reggiani, A., Nijkamp, P. (eds): Spatial Dynamics, Networks and Modelling. Edward Elgar, Cheltenham (2006)

Reggiani, A., Vinciguerra, S.: Network connectivity models: an overview and empirical applications. In: Friesz, T. (ed.) Network Science, Nonlinear Science and Infrastructure Systems. Springer-Verlag, New York (2007)

Reynolds-Feighan, A.J.: EC and US air freight markets: network organisation in a deregulated environment. Transp. Rev. 14, 193-217 (1994)

Reynolds-Feighan, A.J.: The impact of US airline deregulation on airport traffic patterns. Geogr. Anal. 30, 234-253 (1998)

Reynolds-Feighan, A.J.: Traffic distribution in low-cost and full service carrier network in the US air transport market. J. Air Transp. Manag. 7, 265-275 (2001)

Rietveld, P., Brons, M.: Quality of hub-and-spoke networks; the effects of timetable coordination on waiting time and rescheduling time. J. Air Transp. Manag. 7, 241-249 (2001)

Schintler, L.A., Gorman, S.P., Reggiani, A., Patuelli, R., Nijkamp, P.: Small-world phenomena in communication networks: a cross-Atlantic comparison. In: Reggiani, A., Schintler, L.A. (eds) Methods and Models in Transport and Telecommunications: Cross Atlantic Perspectives. Springer-Verlag, Berlin (2005a)

Schintler, L.A., Gorman, S.P., Reggiani, A., Patuelli, R., Gillespie, A., Nijkamp, P., Rutherford, J.: Complex network phenomena in telecommunication systems. Netw. Spat. Econ. 5, 351$370(2005 b)$

Toh, R.S., Higgins, R.G.: The impact of hub-and-spoke network centralization and route monopoly on domestic airline profitability. Transp. J. 24, 16-27 (1985)

Veldhuis, J., Kroes, E.: Dynamics in relative network performance of the main European hub airports. European Transport Conference, Cambridge (2002)

Waters, N.: Network and nodal indices. Measures of complexity and redundancy: a review. In: Reggiani, A., Nijkamp, P. (eds) Spatial Dynamics, Networks and Modelling. Edward Elgar, Cheltenham (2006)

Watts, D.J., Strogatz, S.H.: Collective dynamics of small world networks. Nat. 393, 440-442 (1998)

$\mathrm{Xu}$, J.: How has the literature on the Gini index evolved on the past 80 years? Department of Economics, Dalhousie University Halifax.

http://economics.dal.ca/RePEc/dal/wparch/howgini.pdf (2004) 


\section{Annex A}

In this annex, we will present the top ten scores of the airports - according to the main topological indices illustrated in Table 1 - belonging to the four airline networks A1, A2, B1 and B2. These networks are visualized in the subsequent Annex B.

Table A1 Top-ten scores of airports according to the degree index (corresponding values in brackets)

\begin{tabular}{llll}
\hline A1 & A2 & B1 & B2 \\
\hline MUC (82) & FRA (138) & FRA (106) & FRA (183) \\
FRA (81) & MUC (100) & MUC (105) & MUC (179) \\
DUS (39) & DUS (41) & BRE (97) & HAM (172) \\
HAM (24) & HAM (24) & HAM (97) & DUS (171) \\
STR (18) & STR (18) & BSL (94) & STR (168) \\
TXL (10) & TXL (10) & DUS (94) & LEJ (166) \\
CDG (8) & CDG (8) & LEJ (92) & ZRH (165) \\
NUE (8) & NUE (8) & NUE (92) & TXL (164) \\
BRU (7) & BRU (7) & STR (92) & NUE (163) \\
LHR (6) & MXP (6) & CGN (89) & BRE (162) \\
\hline
\end{tabular}

Table A2 Top-ten scores of airports according to the closeness index (corresponding values in brackets)

\begin{tabular}{llll}
\hline A1 & A2 & B1 & B2 \\
\hline MUC $(0.78)$ & FRA $(0.79)$ & FRA $(0.96)$ & BRE $(1)$ \\
FRA $(0.76)$ & MUC $(0.64)$ & MUC $(0.95)$ & DUS $(1)$ \\
DUS $(0.60)$ & DUS $(0.53)$ & HAM $(0.89)$ & ZRH $(1)$ \\
HAM $(0.55)$ & HAM $(0.51)$ & DUS $(0.87)$ & FRA $(0.98)$ \\
STR $(0.54)$ & STR $(0.50)$ & NUE $(0.86)$ & MUC $(0.95)$ \\
TXL $(0.51)$ & CDG $(0.49)$ & STR $(0.86)$ & HAM $(0.93)$ \\
CDG $(0.51)$ & NUE $(0.49)$ & LEJ $(0.85)$ & STR $(0.91)$ \\
NUE $(0.51)$ & BRU $(0.48)$ & CGN $(0.84)$ & LEJ $(0.89)$ \\
LHR $(0.51)$ & LHR $(0.48)$ & TXL $(0.84)$ & NUE $(0.89)$ \\
MXP $(0.51)$ & MXP $(0.48)$ & ZRH $(0.84)$ & FMO $(0.85)$ \\
& VIE $(0.48)$ & & \\
\hline
\end{tabular}


20 Aura Reggiani, Sara Signoretti, Peter Nijkamp, Alessandro Cento

Table A3 Top-ten scores of airports according to the betweenness index (corresponding values in brackets)

\begin{tabular}{llll}
\hline A1 & A2 & B1 & B2 \\
\hline MUC $(0.51)$ & FRA $(0.76)$ & MUC $(0.06)$ & MUC $(0.06)$ \\
FRA $(0.50)$ & MUC $(0.03)$ & FRA $(0.06)$ & FRA (0.06) \\
DUS (0.06) & DUS $(0.03)$ & DUS $(0.05)$ & DUS $(0.06)$ \\
KUF $(0.05)$ & BKK $(0.02)$ & HAM $(0.05)$ & BRE $(0.05)$ \\
HAM $(0.03)$ & KUF $(0.02)$ & STR $(0.05)$ & CGN $(0.05)$ \\
GOJ $(0.02)$ & HAM $(0.01)$ & BRE $(0.04)$ & HAM $(0.05)$ \\
STR $(0.01)$ & CAI $(0.01)$ & HAJ $(0.04)$ & NUE $(0.05)$ \\
CDG $\left(4.5 \mathrm{e}^{-4}\right)$ & CAN $(0.01)$ & NUE $(0.04)$ & STR $(0.05)$ \\
CGN $\left(9.5 \mathrm{e}^{-5}\right)$ & GOJ $(0.01)$ & TXL $(0.04)$ & ZRH $(0.05)$ \\
BRU $\left(1.9 \mathrm{e}^{-5}\right)$ & GRU $(0.01)$ & CGN $(0.04)$ & CGN $(0.05)$ \\
& JED $(0.01)$ & & DRS $(0.05)$ \\
& KRT $(0.01)$ & & LEJ $(0.05)$ \\
& LOS $(0.01)$ & & \\
\hline
\end{tabular}

Table A4 Top-ten scores of airports according to the clustering coefficient (corresponding values in brackets)

\begin{tabular}{llll}
\hline A1 & A2 & B1 & B2 \\
\hline MUC (0.82) & FRA (0.75) & FRA (0.96) & BRE (1) \\
FRA (0.80) & MUC (0.48) & MUC (0.89) & DUS (1) \\
DUS (0.24) & DUS (0.11) & LEJ (0.77) & ZRH (1) \\
HAM (0.10) & HAM (0.04) & ZRH (0.67) & FRA (0.96) \\
STR (0.06) & STR (0.02) & BSL (0.66) & MUC (0.88) \\
CDG (0.01) & TXL (6e-3) & STR (0.57) & LEJ (0.84) \\
TXL (0.01) & CDG (5e-6) & DUS (0.55) & BSL (0.81) \\
NUE (9e-3) & NUE (4e-3) & HAM (0.55) & GVA (0.67) \\
BRU (6e-3) & BRU (2e-3) & GVA (0.48) & HAM (0.63) \\
MXP (4e-4) & ZRH (2e-3) & TXL (0.47) & STR (0.60) \\
VIE (4e-4) & & & \\
\hline
\end{tabular}


Table A5 Nomenclature of airports under study

\begin{tabular}{ll}
\hline BKK & Bangkok \\
\hline BRE & Bremen \\
BRU & Bruxelles \\
BSL & Basel \\
CDG & Paris Charles de Gaulle \\
CGN & Koln \\
DRS & Dresden \\
DUS & Dusseldorf \\
FMO & Munster \\
FRA & Frankfurt \\
GOJ & Novgorod \\
GRU & Sao Paulo \\
GVA & Geneva \\
HAM & Hamburg \\
JED & Jedda \\
KRT & Khartoum \\
KUF & Samara \\
LEJ & Leipzig \\
LHR & London-Heathrow \\
LOS & Laos \\
MUC & Munich \\
MXP & Milano-Malpensa \\
NUE & Nuremberg \\
PHC & Port Harcour \\
STR & Stuttgart \\
TXL & Berlin-Tegel \\
VIE & Wien \\
ZRH & Zurich \\
\hline &
\end{tabular}




\section{Annex B}

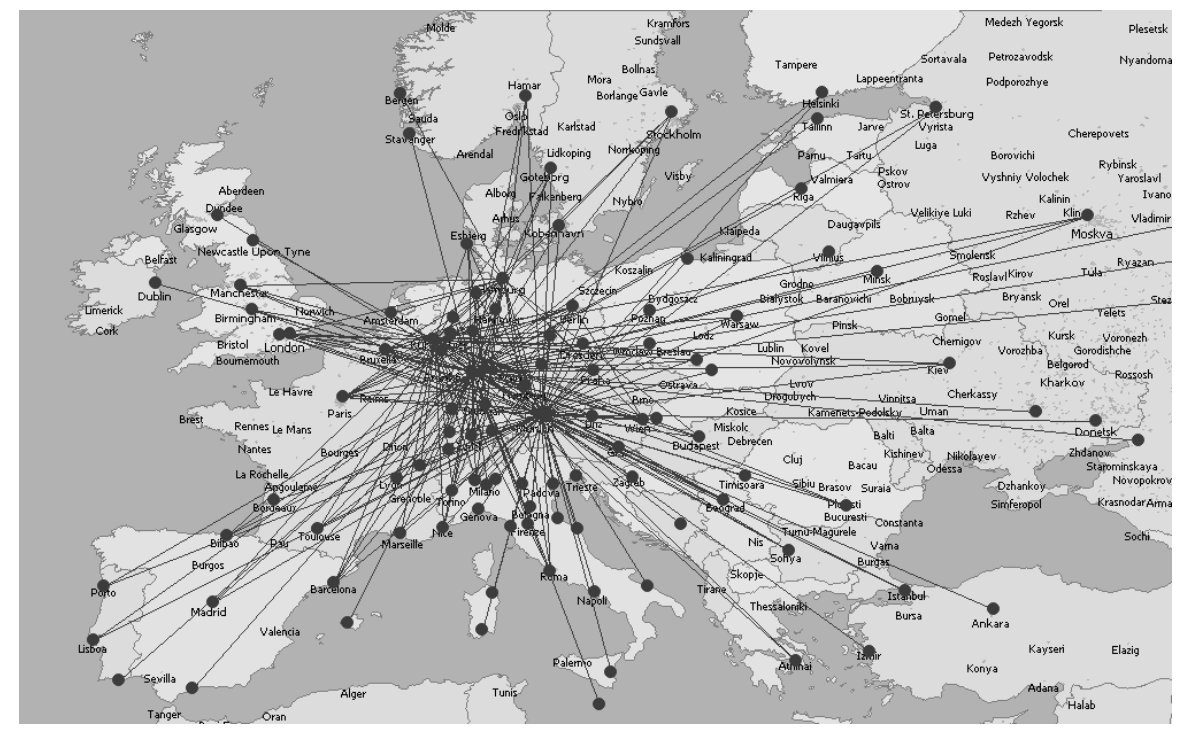

Source: OAG (2006).

Fig. B1 Lufthansa medium haul network in Europe (Network A1)

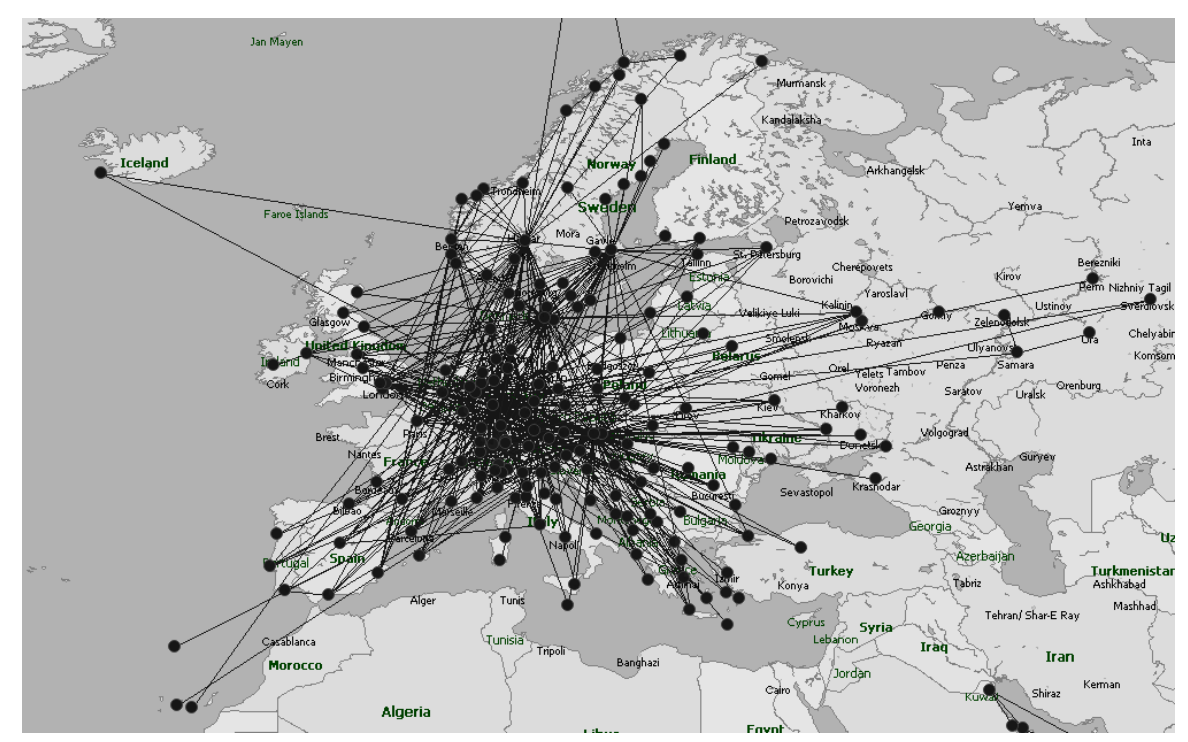

Source: OAG (2006).

Fig. B2 STAR Alliance medium haul network in Europe (Network B1) 


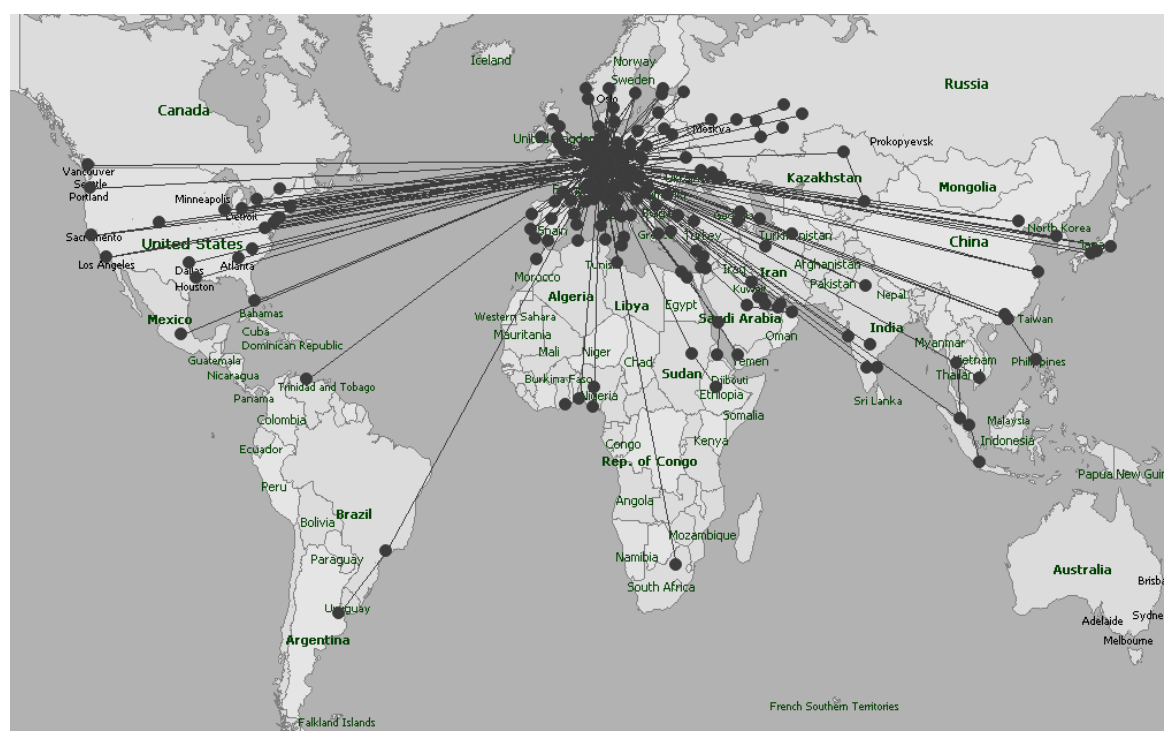

Source: OAG (2006).

Fig. B3 Lufthansa global network (Network A2)

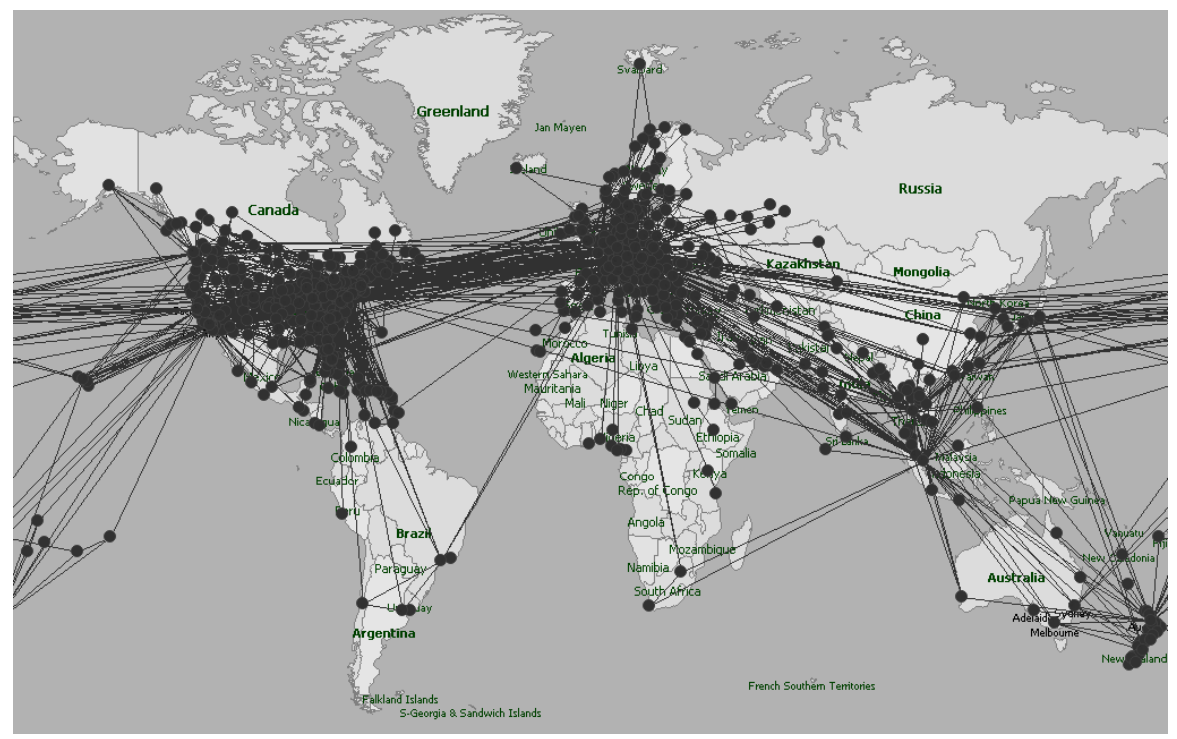

Source: OAG (2006).

Fig. B4 STAR Alliance global network (Network B2) 